\title{
CONSTITUINTES QUÍMICOS DE Zanthoxylum ekmanii (URB.) ALAIN
}

\author{
Valdir Alves Facundo* e Augusto Sérgio Pinto da Silveira \\ Departamento de Química, Universidade Federal de Rondônia, Br 364 km 9,5, 78900-500 Porto Velho - RO \\ Raimundo Braz Filho
}

Setor de Química de Produtos Naturais, Centro de Ciências e Tecnologia, Universidade Estadual do Norte Fluminense, Av. Alberto

Lamego, 2000, 28015-620 Campos - RJ

Angelo C. Pinto e Claudia M. Rezende

Instituto de Química, Universidade Federal do Rio de Janeiro, Centro de Tecnologia, Bloco A, Cidade Universitária, 21945-970

Rio de Janeiro - RJ

Recebido em 17/2/04; aceito em 9/9/04; publicado na web em 17/2/05

\begin{abstract}
CHEMICAL CONSTITUENTS OF Zanthoxylum ekmanii (URB.) ALAIN. Chemical investigation of Z. ekmanii resulted in the isolation of skimmianine, dictamnine, tembamide, sesamin, lupeol and $\beta$-sitosterol. The structures were established by spectroscopic analyses. This is the first report on the phytochemical study of the roots and leaves of Z. ekmanii .
\end{abstract}

Keywords: Zanthoxylum ekmanii; Rutaceae; alkaloids.

\section{INTRODUÇÃO}

O gênero Zanthoxylum (Rutaceae) compreende mais de 200 espécies e encontra-se distribuído em todo mundo ${ }^{1}$. Investigações fitoquímicas anteriores de espécies deste gênero revelaram a presença de alcalóides, flavonóides, cumarinas, lignanas e terpenos ${ }^{2-5}$. Várias destas espécies são utilizadas na medicina popular no tratamento de doenças cardiovasculares, tuberculose, malária, para aliviar dor de dente e contra mordida de cobra $^{6-9}$. O estudo fitoquímico de duas espécies comuns do nordeste do Brasil, Z. syncarpum e Z. rugosum, conduziu ao isolamento dos metabólitos secundários skimianina, cis$N$-metilcanadina, isopimpinelina, xantotoxina, lupeol, ácido centipédico, $3 \beta$-O- $\beta$-D-glucopiranosil-sitosterol, 6-cantinona, 6,7-dimetoxicumarina, 6,7,8-trimetoxicumarina, avicenina, hesperidina, ácido 3- $(9 \mathrm{H}-$ $\beta$-carbolina-1-il)-(Z)-2-propenóico e 2-hidróxi-2-(4-hidroxifenil)etil (trimetil) amônio ${ }^{10,11}$.

A espécie Z. ekmanii, conhecida popularmente como "mamica de porca", é uma árvore de 7-13 m de altura, possuindo acúleos na base do tronco e cujo chá das folhas e raízes é utilizado, principalmente pela população do baixo Madeira, Porto Velho - Rondônia, no tratamento da malária, em lavagens vaginais e para aliviar dor de dente. O estudo do óleo essencial de suas folhas apresentou como constituintes majoritários o germacreno D $(16,0 \%)$ e $(E)$ - $\beta$-cariofileno $(15,5 \%)^{12}$. O presente trabalho tem como objetivo descrever o estudo fitoquímico das folhas e raízes de $Z$. ekmanii.

\section{RESULTADOS E DISCUSSÃO}

As estruturas dos alcalóides skimianina (1) e dictamina (2) foram definidas com base na análise dos espectros de RMN de ${ }^{1} \mathrm{H}$ e de ${ }^{13} \mathrm{C}$ uni e bidimensionais e por comparação com valores de $\mathrm{RMN} \mathrm{de}{ }^{13} \mathrm{C}$ descritos na literatura ${ }^{13}$. Dois dubletos observados no espectro de $\mathrm{RMN}$ de ${ }^{1} \mathrm{H}$ de 1 [ $\delta_{\mathrm{H}} 7,53,(J=2,9 \mathrm{~Hz}, \mathrm{H}-10)$ e $\left.6,99(J=2,9 \mathrm{~Hz}, \mathrm{H}-11)\right]$ e de $2\left[\delta_{\mathrm{H}} 7,55(J=2,8 \mathrm{~Hz}, \mathrm{H}-10)\right.$ e $\left.6,98(J=2,8 \mathrm{~Hz}, \mathrm{H}-11)\right]$, correlacionados nos espectros HMQC com os sinais de átomos de

*e-mail: vfacundo@unir.br<smiles></smiles>

$1 \mathrm{R}=\mathrm{OCH}_{3}$

$2 \mathrm{R}=\mathrm{H}$<smiles>COc1ccc([C@@H](O)CNC(=O)c2ccccc2)cc1</smiles>

3

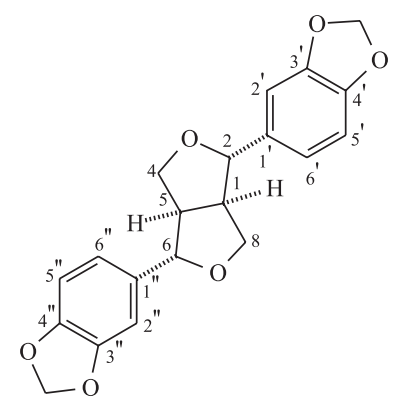

4<smiles>C=C(C)[C@@H]1CCC2(C)CC[C@H]3C(CCC4[C@@]3(C)CCC3C(C)(C)C(O)CC[C@@]34C)C12</smiles>

5<smiles>CCC(CC[C@H](C)[C@H]1CC[C@H]2[C@H]3CC=C4CC(O)CC[C@]4(C)[C@H]3CC[C@@]21C)C(C)C</smiles>

carbono em $\delta_{\mathrm{C}} 142,9$ (C-10) e 104,6 (C-11) de 1 e 143,8 (C-10) e 105,0 (C-11) de 2, confirmaram a presença de anel furânico 1,2dissubstituído em ambas as substâncias. A comparação dos espectros de $\mathrm{RMN}$ de ${ }^{1} \mathrm{H}$ e de ${ }^{13} \mathrm{C}$ de $\mathbf{1}$ e 2 mostrou que a principal diferença entre os dois alcalóides é o padrão de substituição do anel benzênico. $\mathrm{O}$ 
espectro de $\mathrm{RMN}$ de ${ }^{1} \mathrm{H}$ de $\mathbf{1}$ revelou a presença de dois grupos metoxila adicionais em relação a $\mathbf{2}$.

A estrutura da tembamida (3) foi caracterizada através dos espectros de $\mathrm{RMN}$ de ${ }^{1} \mathrm{H}$ e de ${ }^{13} \mathrm{C}$ uni e bidimensionais, por comparação com valores de $\mathrm{RMN}$ de ${ }^{13} \mathrm{C}$ descritos na literatura ${ }^{14}$ e por CG-EM, que apresentou o íon $\mathrm{m} / z, 253\left(20 \%, \mathrm{C}_{16} \mathrm{H}_{15} \mathrm{O}_{2} \mathrm{~N}\right)$ o qual, adicionado de uma molécula de $\mathrm{H}_{2} \mathrm{O}$, permitiu a dedução da fórmula molecular de 3 como $\mathrm{C}_{16} \mathrm{H}_{17} \mathrm{O}_{3} \mathrm{~N}$. Um sistema AA'BB' foi observado no espectro de $\mathrm{RMN}$ de ${ }^{1} \mathrm{H}$ com $J=8,8 \mathrm{~Hz}$, sugerindo a presença de um anel aromático para-dissubstituído contendo um grupo metoxila. O sistema envolvendo o grupo $N$-metilênico ligado ao carbono metínico carbinólico foi caracterizado pelos sinais em $\delta_{\mathrm{H}} 3,88$ (ddd, $J=14,3,7,7$ e 3,3 Hz), 3,51 (ddd, $J=14,3,8,0$ e 4,8 Hz) e 4,91 (dd, $J=7,7$ e 4,8 Hz). $\mathrm{O}$ espectro de $\mathrm{RMN}$ de ${ }^{13} \mathrm{C}$ revelou o sinal da carbonila amídica aromática em $\delta_{\mathrm{C}} 168,5$

A caracterização estrutural da sesamina (4) baseou-se na análise dos espectros de $\mathrm{RMN}$ de ${ }^{1} \mathrm{H}$ e de ${ }^{13} \mathrm{C}$ uni e bidimensionais e na comparação com dados de $\mathrm{RMN}$ de ${ }^{1} \mathrm{H}$ e de ${ }^{13} \mathrm{C}$ descritos na literatura ${ }^{15}$.

As estruturas do lupeol (5) e do $\beta$-sitosterol (6) foram identificadas através dos dados fornecidos pelos espectros de $\mathrm{RMN}$ de $\mathrm{H}^{1}$ e de ${ }^{13} \mathrm{C}$, comparados com valores descritos na literatura ${ }^{16}$.

Uma busca rápida na literatura pôde registrar a atividade biológica das substâncias identificadas no extrato de Z. ekmanii. A skimianina (1) possui atividade cardiovascular ${ }^{17}$ e leishimanicida ${ }^{18}$. A dictamina (2) inibe a agregação plaquetária ${ }^{19}$. A lignana sesamina (4) apresenta atividade antifúngica ${ }^{20}$ e efeito anti-hipertensivo ${ }^{21}$ e o lupeol (5) apresenta, in vitro, ação inibitória contra o protozoário Plasmodium falciparum ${ }^{22}$, o que justifica o uso etnobotânico da espécie em estudo.

\section{PARTE EXPERIMENTAL}

\section{Procedimentos experimentais gerais}

Os espectros de RMN foram obtidos nos aparelhos Bruker Avance $500\left({ }^{1} \mathrm{H}: 500 \mathrm{MHz} ;{ }^{13} \mathrm{C}: 125 \mathrm{MHz}\right)$ e Jeol - $400\left({ }^{1} \mathrm{H}: 400\right.$ $\left.\mathrm{MHz} ;{ }^{13} \mathrm{C}: 100 \mathrm{MHz}\right)$. Os espectros de massas foram registrados por impacto eletrônico $(70$ eV) em CG-EM Hewlett - Packard 5971 usando coluna capilar (30 $\mathrm{m}$ x 0,25 $\mathrm{mm} \times 0,25 \mathrm{~mm}$ ) dimetilpolisiloxano $\mathrm{BD}-1$, He como gás de arraste (fluxo de $1 \mathrm{~mL} / \mathrm{min}$ ) e $\mathrm{T}_{\text {inj }} 250{ }^{\circ} \mathrm{C}$ (modo com divisão de fluxo); programação do forno cromatográfico: $7 \%$ min entre $35-180{ }^{\circ} \mathrm{C}$ e $10{ }^{\circ} \mathrm{C} /$ min entre $180-250{ }^{\circ} \mathrm{C}$ na coluna. Nas separações cromatográficas em coluna aberta usou-se sílica gel (Merck, 60-230 mesh). As placas cromatográficas foram reveladas com luz UV ( $\left.\lambda_{\max } 254 \mathrm{~nm}\right)$, vapores de iodo e/ou solução alcoólica de vanilina e ácido sulfúrico.

\section{Planta}

As folhas e raízes de Z. ekmanii foram coletadas no estado de Rondônia, no sudoeste da Floresta Amazônica, Brasil, em março de 2000. A identificação botânica foi feita no Instituto Nacional de Pesquisa da Amazônia (INPA) e uma exsicata encontra-se depositada no herbário da Universidade Federal de Rondônia, Rondônia, Brasil, sob o número 041.

\section{Extração e isolamento}

As raízes secas e trituradas $(2,0 \mathrm{~kg}$ ) foram extraídas com etanol ( 3 L x 3 ) a temperatura ambiente. O solvente foi destilado sob pressão reduzida e forneceu 41,0 g de uma massa de coloração marrom. Parte deste material $(35,0 \mathrm{~g})$ foi adsorvido em sílica gel $(90,0 \mathrm{~g})$ e a mistura, sob a forma de pastilha, colocada em uma coluna cromatográfica e eluída com hexano, clorofórmio, acetato de etila e metanol. A fração clorofórmica $(9,3 \mathrm{~g})$ foi novamente submetida à cromatografia em coluna de gel de sílica e eluida com misturas de hexano e clorofórmio em polaridade crescente, obtendo-se 83 frações. As frações de 10 a 19 foram purificadas por recristalização, obtendose $\beta$-sitosterol (6, 23,4 mg), lupeol (5, 43,7 mg), dictamina (2,31,0 mg), tembamida $(\mathbf{3}, 14,9 \mathrm{mg})$ e skimmianina $(\mathbf{1}, 11,1 \mathrm{mg})$.

O extrato etanólico $(19,0 \mathrm{~g})$ das folhas secas e trituradas (400 g) foi submetido ao mesmo procedimento utilizado para as raízes. A fração clorofórmica foi cromatografada em coluna de gel de sílica e eluida com misturas de hexano e clorofórmio em polaridade crescente, fornecendo 32 frações. As frações 7, 11 e 21, após serem recromatografadas, forneceram $\beta$-sitosterol $(\mathbf{6}, 18,0 \mathrm{mg})$, sesamina $(\mathbf{4}, 27,4 \mathrm{mg})$ e skimianina $(\mathbf{1}, 37,0 \mathrm{mg})$.

\section{AGRADECIMENTOS}

Os autores agradecem ao CNPq, BASA e FAPERJ pelas bolsas e apoios financeiros concedidos e ao Dr. C. Ferreira, do INPA, pela identificação botânica da planta.

\section{REFERÊNCIAS}

1. Talapatra, S. K.; Dutta, S. K.; Talapatra, B.; Phytochemistry 1973, 12, 729.

2. Frank, R. S.; Iraj, A. S.; Phytochemistry 1977, 16, 2003.

3. Fish, F.; Waterman, P. G.; Phytochemistry 1972, 11, 3007.

4. Diehl, E. E.; von Poser, G. L.; Henriques, A. T.; Biochem. Syst. Ecol. 2000, 28, 275.

5. Facundo, V. A.; Tese de Doutorado, Universidade Federal do Ceará, Brasil, 1999.

6. Calderwood, J. M.; Finkelstein, N.; Fish, F.; Phytochemistry 1970, 9, 675.

7. Gessler, M. C.; Nkunya, M. H. H.; Mwasumbi, L. B.; Heinrich, M.; Tanner, M.; Acta Tropica 1994, 56, 65.

8. Weenen, H.; Nkunya, M. H. H.; Bray, D. H.; Mwasumbi, L. B.; Kinabo, L. S.; Kilimali, V. A. E. B.; Wilnberg, J. B. P. A.; Planta Med. 1990, 56, 371 .

9. Arruda, M. S. P.; Fernandes, J. B.; Vieira, P. C.; Silva, M. F. G. F.; Pirani, J. R.; Biochem. Syst. Ecol. 1992, 20, 173.

10. Facundo, V. A.; Morais, S. M.; Souza, R. T.; Braz-Filho, R.; Rev. Latinoamer. Quim. 2002, 30, 61.

11. Facundo, V. A.; Morais, S. M.; Braz-Filho, R.; Rev. Bras. Farm. 1999, 78, 57.

12. Facundo, V. A; Rezende, C. M.; Pinto, A. C.; Morais, S. M.; J. Essent. Oil Res. 2003, 15, 402

13. Akonda, A.; Picot, F.; Potier, P.; Poupat, C.; Sévenet, T.; Phytochemistry 1978, 17, 166.

14. Patra, A.; Mitra, A. K.; Ghosh, A.; Mukhopadhyay, P. K.; Org. Mag. Res. 1981, 16, 65.

15. Andrew, P.; Ward, R. S.; Rao, V. E.; Sartry, K. V.; Tetrahedron 1976, 32, 2783.

16. Reynolds, W. F.; McLean, S.; Poplawski, J.; Tetrahedron 1986, 42, 3419.

17. Cheng, J. T.; Chang, S. S.; Chen, I. S.; Arch. Int. Pharmacodyn. Ther. 1990, 306,65 .

18. Fournet, A.; Barrios, A. A.; Munoz, V.; Hocquemiller, R.; Cave, A.; Bruneton, J.; Antimicrob. Agents Chemother. 1993, 37, 859.

19. Chen, I. S.; Lin, Y. C.; Tsai, I. L.; Teng, C. M.; Ko, F. N.; Ishikawa, T.; Ishii, H.; Phytochemistry 1995, 39, 1091.

20. Jayasinghea, L.; Kumarihamya, B. M. M.; Jayarathna, K. H. R. N.; Udishani, N. W. M.; Bandara, B. M. R.; Hara, N.; Fujimoto, Y.; Phytochemistry 2003, 62, 637.

21. Nakano, D.; Itoh, C.; Takaoka, M.; Kiso, Y.; Tanaka, T.; Matsumura. Y.; Biol. Pharm. Bull. 2002, 25, 1247.

22. Ziegler, H. L.; Staerk, D.; Christensen, J.; Hviid, L.; Hagerstrand, H.; Jaroszewski, J. W.; Antimicrob. Agents Chemother. 2002, 46, 144. 